\title{
Liderança, compatibilidade treinador-atleta e satisfação no andebol: percepção de atletas novatos e experientes
}

\author{
Rui Gomes' - Universidade do Minho, Braga, Portugal \\ Paulo Paiva - Universidade do Minho, Braga, Portugal
}

\begin{abstract}
Resumo
Este trabalho compara a percepção de atletas em formação desportiva (novatos) e em competição adulta (experientes), ao nível dos estilos de liderança dos treinadores, da compatibilidade com os treinadores e da satisfação com a liderança. Participaram 91 atletas do sexo masculino de andebol, incluídos na formação desportiva ( $\mathrm{n}=59$; "novatos") e competição ( $\mathrm{n}=32$; "experientes"). Foram usadas as seguintes medidas: Questionário Demográfico, Escala Multidimensional de Liderança no Desporto-2, Medida de Compatibilidade Treinador-Atleta e Questionário de Satisfação em Atletas. Devemos realçar dois resultados principais: os novatos avaliaram mais positivamente os estilos de liderança dos treinadores, evidenciaram maior compatibilidade treinadoratleta, e assumiram maior satisfação com a liderança; e as dimensões da liderança explicaram $32 \%$ da variância associada à compatibilidade treinador-atleta nos novatos e $43 \%$ nos experientes.

Palavras-chave: Liderança, Satisfação, Compatibilidade treinador-atleta, Tempo de prática desportiva.
\end{abstract}

Leadership, coach-athlete compatibility and satisfaction in handball: perceptions of novice and experienced athletes

\begin{abstract}
This work compares the perceptions of two groups of athletes (novice and experienced athletes) about the leadership styles of their coaches, compatibility with the coach and satisfaction with leadership. The sample included 91 male athletes of a handball team; 59 were youth sport athletes (novice) and 32 were adult sport athletes (experienced). We applied the following measurements: Demographic Questionnaire, Multidimensional Scale of Leadership in Sports, Coach-Athlete Compatibility, and Athlete Satisfaction Questionnaire. The results showed that: novice athletes evaluated more positively the leadership styles, assumed more compatibility with the coach, and demonstrated more satisfaction with leadership. Therefore, leadership scales explained $32 \%$ of the variance related with Coach-Athlete Compatibility in novice athletes and $43 \%$ in experienced athletes.

Keywords: Leadership, Satisfaction, Coach-athlete compatibility, Sports experience.
\end{abstract}

A liderança tem sido alvo de vários estudos por parte da Psicologia, estimando-se que até 2001 tenham sido publicados mais de 3.500 trabalhos neste domínio (Weinberg \& Gould, 2007). Em termos desportivos, uma das propostas que mais contributos deu nesse sentido foi o modelo multidimensional de liderança no desporto (Chelladurai, 1993; Chelladurai \& Saleh, 1978, 1980).

Nesse caso, preconiza-se que a compreensão da liderança no desporto deve considerar os factores antecedentes (e condicionadores) da acção dos treinadores, nomeadamente, as características da situação, do próprio treinador e dos atletas. A título de exemplo, o técnico deve atender à situação de estar a treinar equipas de recreação ou de alta competição (características da situação), analisar quais os desejos e expectativas dos membros da equipa (características dos atletas) e quais são os seus objectivos pessoais e maneira de ser (características do próprio treinador). Esses aspectos vão influenciar os comportamentos efectivos dos treinadores na gestão das equipas e dos atletas. Assim, os comportamentos actuais assumidos são influenciados pelas características do próprio treinador, pelos comportamentos desejados e preferidos dos atletas e pelos comportamentos

\footnotetext{
1 E-mail: rgomes@psi.uminho.pt
}

“exigidos" ou requeridos pela situação.

A partir daqui, é avançada como hipótese principal a possibilidade de o líder desportivo conseguir maiores níveis de rendimento e satisfação nos atletas e respectivas equipas, quando alcança uma maior congruência entre os seus comportamentos actuais, os preferidos pelos atletas e os requeridos pela situação. Na prática, isso significa que o treinador deve estar atento às necessidades dos atletas $\mathrm{e}$ às particularidades do seu contexto de acção, uma vez que, se conseguir atender a esses aspectos na sua acção, terá maior probabilidade de atingir sucesso desportivo e promover experiências positivas nos praticantes. Os dados da investigação têm vindo a demonstrar uma relação positiva entre a congruência dos comportamentos dos treinadores e os resultados desportivos alcançados e a maior satisfação dos atletas (Chelladurai, 1984a; Horne \& Carron, 1985; Riemer \& Chelladurai, 1995; Weiss \& Friedrichs, 1986). De igual modo, esses efeitos parecem também extensíveis à maior orientação para a realização das tarefas por parte dos atletas (Gardner, Shields, Bredmeier \& Bostrom, 1996; Pease \& Kozub, 1994; Westre \& Weiss, 1991) bem como ao tipo de avaliações e atitudes que manifestam acerca dos treinadores (Chelladurai, 1984b).

Apesar do valor inegável dessa proposta, um dos aspectos mais deficitários prende-se com a não- 
consideração dos contributos mais recentes do estudo da liderança, nomeadamente dos modelos resultantes da liderança transformacional (Bryman, 1992), visionária (Sashkin, 1988) ou carismática (Conger, 1989). Esses autores centraram-se na observação de líderes que conseguiram produzir mudanças extraordinárias (ou acima do esperado) nos membros do grupo, levando-os a comprometerem-se com os objectivos do grupo e sacrificando-se pessoalmente em nome de um bem colectivo (Bass, 1985; Conger \& Kanungo, 1987). Esses avanços vêm mais tarde a ser incorporados no modelo multidimensional, procurando-se assim fazer a ligação com a liderança transaccional já existente na proposta. Assim, para além dos aspectos mais relacionais entre treinador e atletas, onde a interacção entre ambos se baseia numa "transacção" entre algo que o técnico pode oferecer e algo que os atletas desejam (ex: recompensas, elogios, status, desportivo, etc.), propõe-se também que nesse processo possa existir uma mudança de ideias, valores e princípios nos atletas, como resultado da acção transformacional dos treinadores. Desse ponto de vista, o modelo sofre uma alteração significativa, tornando-se a liderança transformacional um factor antecedente, que pode influenciar as características do líder, dos membros do grupo e da própria situação (Riemer, 2007).

As razões para atender a esses avanços mais recentes do estudo da liderança são evidentes quando se verifica que as pessoas orientadas por líderes transformacionais, carismáticos ou visionários (consoante a abordagem em causa) alcançam maiores níveis de eficácia pessoal e de rendimento e percepcionam experiências mais positivas de satisfação, comprometimento e coesão (Avolio \& Bass, 1988; Bass, 1990; Bass \& Avolio, 1997; Brown \& Dodd, 1999; Podsakoff, MacKenzie, Moorman \& Fetter, 1990).

Assim sendo, a situação actual do estudo da liderança no desporto tem vindo a aproximar-se cada vez mais dos contributos contemporâneos do estudo deste tema na psicologia geral, mas apesar disso são escassos os estudos que incluam essas dimensões na análise dos líderes desportivos. Isso mesmo é reconhecido pelo próprio Chelladurai (2007) ao registar uma insuficiência de trabalhos centrados no estudo das características transformacionais dos treinadores e da relação com variáveis pessoais e desportivas dos atletas. Um dos aspectos que mais pode contribuir para essa situação, prende-se com a circunstância das metodologias de avaliação da liderança no desporto não incorporarem essas dimensões na análise do trabalho dos treinadores. Obviamente, esta lacuna torna muito difícil o avanço dos conhecimentos nesta área, impossibilitando a comprovação do interesse da liderança transformacional em contextos desportivos.

Foi a partir das vantagens inquestionáveis do modelo multidimensional de liderança no desporto e da necessidade de analisarmos a importância da liderança transformacional na acção dos treinadores que este trabalho foi organizado. Assim, procurámos analisar a percepção dos estilos de liderança de atletas de andebol, observando-se também a relação com os níveis de compatibilidade entre treinador e atletas e com a satisfação com a liderança.

No que se refere à relação da liderança com as outras duas variáveis em análise neste trabalho, alguns aspectos devem ser realçados. Assim, o estudo da percepção de compatibilidade entre os atletas e os respectivos treinadores não tem merecido a devida atenção por parte dos investigadores, isto apesar de se aceitar que esse factor pode ter uma influência no modo como os atletas avaliam e reagem às acções dos respectivos técnicos (Kenow \& Williams, 1999). Alguns dos resultados encontrados por esses autores, demonstram que os atletas que evidenciam maior compatibilidade com os treinadores tendem a relatar menos efeitos negativos do ponto de vista cognitivo e emocional aos comportamentos assumidos pelos técnicos em situações de competição. De igual modo, maior percepção de compatibilidade tende a correlacionar-se com avaliação mais positiva dos níveis de apoio fornecidos pelos treinadores e a uma melhor comunicação entre as partes. Apesar do interesse destes resultados, existe ainda uma escassez de conhecimento sobre a relação com os estilos de liderança, particularmente com as dimensões transformacionais da acção dos treinadores.

Quanto à relação entre o exercício da liderança e a satisfação, o cenário é bastante inverso, uma vez que este tema tem merecido uma ampla análise por parte das ciências sociais (Riemer \& Chelladurai, 1998). Nesse caso, existem dados sobre a relação da satisfação dos membros do grupo/organização e diferentes indicadores pessoais e profissionais, nomeadamente os níveis de esforço demonstrado na execução das tarefas, a vontade de permanecer no grupo/organização, a disponibilidade para colaborar com os restantes elementos da equipa e os níveis de bem-estar e realização pessoal (Saal \& Knight, 1988). Do ponto de vista desportivo, como referem Chelladurai e Riemer (1997), a satisfação deve ser entendida com um estado afectivo positivo que resulta de uma avaliação dos atletas acerca das estruturas, processos e resultados obtidos na actividade atlética. Um desses factores refere-se à satisfação dos atletas com a liderança no desporto, tendo sido esta a dimensão escolhida para 
avaliação neste estudo. Esta avaliação foi efectuada a partir do desenvolvimento do Questionário de Satisfação em Atletas, que representa uma das medidas mais completas acerca desse fenómeno no desporto. No entanto, tal como no caso da compatibilidade, são ainda deficitários os dados da investigação sobre a relação entre os estilos de liderança (particularmente das facetas transformacionais) e a satisfação dos atletas, sendo esta lacuna tão mais negativa se atendermos à importância que tendemos a atribuir à necessidade dos atletas se sentirem satisfeitos com a actividade desportiva.

Em síntese, este estudo coloca em conjunto três variáveis que têm demonstrado relevância na investigação no âmbito desportivo, procurando clarificar as relações entre elas e o possível impacto de domínios de liderança mais contemporâneos no estudo da acção dos líderes.

A forma como procurámos responder a essas questões baseou-se ainda numa distinção entre atletas mais novos (incluídos em escalões de formação desportiva) e atletas mais velhos (incluídos em sistemas desportivos onde a competição desportiva é um critério mais evidente de avaliação do sucesso). Esta divisão foi realizada não só por escassearem estudos que comparem atletas de diferentes idades quanto à avaliação dos estilos de liderança dos treinadores, mas também pela existência de resultados na literatura que nos alertam para a possibilidade dos objectivos e filosofias subjacentes ao desporto de formação desportiva serem diferentes do desporto de alta competição (Adelino, Vieira \& Coelho, 2000; Gomes, 1997; Martens, 1990; 1996). Apesar da unanimidade em torno desta ideia, a verdade é que pouco se sabe sobre se atletas incluídos em ambos os contextos avaliam realmente de forma diferente os seus treinadores e apresentam experiências pessoais e desportivas distintas. Desse modo, adoptámos as designações "equipas de atletas novatos" para nos referirmos aos atletas mais novos (com idades compreendidas entre os 13 e os 17 anos) e "equipas de atletas experientes" para nos referirmos aos atletas mais velhos (idades acima dos 18 anos). Assim, nas equipas dos atletas novatos foram consideradas os escalões desportivos de iniciados e juvenis, enquanto nos mais experientes considerámos os atletas juniores e seniores. Apesar da "artificialidade" dessa distinção, uma vez que o factor idade nem sempre discrimina a exigência competitiva colocada aos atletas, aceita-se no caso do andebol que os atletas até aos escalões de juvenis estão em "fase de formação desportiva" enquanto o escalão de juniores representa já a preparação para a alta competição, onde estão os atletas seniores. Do ponto de vista da literatura, esta distinção encontra alguma representatividade na divisão proposta por Côté e Hay (2002), ao formularem três etapas no envolvimento desportivo dos atletas: 1) os anos de iniciação e conhecimento, onde as crianças se integram em contextos desportivos organizados e se familiarizam com a prática desportiva; 2) os anos de especialização, onde os jovens se centram mais numa actividade desportiva, aprendendo e desenvolvendo as competências da mesma; e 3) os anos de investimento, onde os jovens se comprometem com a actividade desportiva, procurando obter altos níveis de rendimento e alcançar o status de praticantes de elite. Apesar dos autores não serem muito directivos em termos de idades (até porque este factor depende imenso da modalidade em causa), existe algum acordo sobre a entrada dos jovens em processos de especialização a partir dos 13 anos (Bloom, 1985; Côté, 1999; Côté, Young, North \& Duffy, 2007). No nosso caso, essa distinção parece fazer sentido, uma vez que no escalão de iniciados os jovens começam uma aprendizagem dos conteúdos tácticos (e técnicos) específicos da modalidade, aperfeiçoando essas competências no escalão seguinte (juvenis). Por isso, aceita-se mais a ideia de que no escalão de juniores os atletas não só vão automatizando as competências da modalidade, como vão testando essas capacidades num modelo mais competitivo, onde os resultados desportivos adquirem maior peso e importância.

Tendo em consideração esses aspectos, este estudo procura comparar as diferenças nas experiências desportivas entre, por um lado, jovens em formação desportiva (novatos), e, por outro lado, jovens adultos em modelos de competição desportiva (experientes). Os aspectos analisados prenderam-se com a percepção dos estilos de liderança, a compatibilidade com o treinador e os níveis de satisfação com o exercício do poder por parte dos responsáveis técnicos.

Mais concretamente, procuraram-se atingir os seguintes objectivos:

1) Analisar a relação entre a compatibilidade treinador e atletas, os estilos de liderança e a satisfação dos atletas com a liderança;

2) Analisar as diferenças entre os atletas novatos e experientes, no nível da compatibilidade com o treinador, dos estilos de liderança e da satisfação com a liderança;

3) Analisar as variáveis de liderança preditoras da compatibilidade treinador e atletas nos dois grupos de atletas. 


\section{Método}

\section{Participantes}

Participaram no estudo 91 atletas do sexo masculino de um clube de andebol do norte do país, com idades compreendidas entre os 13 e os 42 anos $(\mathrm{M}=17,44 ; \quad \mathrm{DP}=4,95)$. Em relação ao escalão competitivo, 59 atletas enquadraram-se nas equipas de atletas novatos $(64,8 \%)$ e 32 nas equipas de atletas experientes $(35,2 \%)$. O número de anos de prática desportiva variou entre 1 e 32 anos $(M=6,86$; $\mathrm{DP}=5,18)$ para a amostra total. No caso dos atletas novatos, os anos de prática desportiva variaram entre 1 e 12 anos $(M=4,69 ; \mathrm{DP}=2,96)$. Nos atletas experientes verificou-se uma amplitude de anos de prática entre $3 \mathrm{e}$ $32(\mathrm{M}=10,84 ; \mathrm{DP}=6,02)$. Os anos de trabalho com o actual treinador apontaram uma amplitude de tempo entre 1 e 10 anos $(M=1,75 ; \quad D P=1,40)$. Mais concretamente, nos atletas novatos os anos de trabalho com o actual treinador variaram entre 1 e 12 anos $(M=1,20 ; D P=0,41)$. Nos atletas experientes verificouse uma amplitude de anos de trabalho com o actual treinador entre 1 e 2 anos $(\mathrm{M}=1,59 ; \mathrm{DP}=0,50)$.

\section{Instrumentos}

Foi administrado a todos os atletas que participaram neste estudo um questionário que englobava os seguintes instrumentos de avaliação psicológica:

Questionário Demográfico: avaliou variáveis como a idade, o escalão desportivo, a carreira desportiva dos atletas (e.g., anos de prática e número de internacionalizações) e o percurso desportivo realizado com o actual treinador (e.g., anos de trabalho e títulos alcançados).

Medida de Compatibilidade Treinador-Atleta (MCTA) (Gomes, 2008b). Este instrumento foi desenvolvido a partir dos trabalhos de Kenow e Williams (1999) e Williams e colaboradores (2003). Originalmente, esses autores propuseram apenas um item para avaliar esta dimensão ("eu e o meu treinador(a) somos compatíveis"). No entanto, tal situação não permite uma análise das características psicométricas do instrumento e da relevância estatística do mesmo. No sentido de permitir esse tipo de análises e de introduzir maior "profundidade" à compreensão do fenómeno, foram adicionadas quatro afirmações ao item original do instrumento. A formulação dos itens teve por base o conceito original subjacente a esta medida, incluindose indicadores relacionados com a compatibilidade entre objectivos, ideias, maneira de ser e personalidade entre treinador e atletas. Assim sendo, o instrumento ficou constituído por cinco itens, sendo respondidos numa escala tipo Likert de 9 pontos $(1=\mathrm{Nada}$ compatíveis; 9=Muito compatíveis). Mais concretamente, a escala avaliou a consonância e compatibilidade entre treinador e atletas, em termos desportivos e pessoais. O escore foi calculado por meio da soma dos valores dos itens, dividindo depois esse valor pelo número total de itens da escala. As pontuações mais altas significam valores mais elevados de compatibilidade entre treinador e atletas.

Escala Multidimensional de Liderança no Desporto (EMLD-2) (Gomes, 2008a). Este instrumento foi desenvolvido com o objectivo de avaliar os estilos e comportamentos de liderança assumidos pelos treinadores, sendo constituído por nove dimensões e 55 itens: 1) treino e instrução (9 itens): refere-se aos processos de treino, métodos de ensino e indicações dadas pelos treinadores acerca do que os atletas devem fazer ou como podem corrigir e melhorar as suas capacidades desportivas; 2) respeito e tratamento justo (6 itens): refere-se à tendência do treinador tratar os atletas com equidade e justiça, considerando nas suas decisões a dimensão pessoal e humana dos membros da equipa; 3) apoio social (6 itens): comportamentos do treinador orientados para o bem-estar dos atletas, demonstrando uma preocupação pessoal com os problemas dos membros da equipa, bem como um interesse em desenvolver relações pessoais francas e informais; 4) visão de futuro e optimismo (5 itens): descreve os comportamentos entusiásticos e optimistas do treinador acerca dos objectivos e tarefas a desenvolver, bem como a capacidade de envolver os membros da equipa num ideal positivo de futuro para a equipa; 5) motivação para a realização (6 itens): comportamentos do treinador com o objetivo de promover o desejo de sucesso e esforço contínuo dos atletas na concretização das tarefas e objectivos formulados, assumindo expectativas elevadas de realização para todos os membros da equipa; 6) feedback positivo (6 itens): comportamentos de reforço e reconhecimento do treinador em face do bom rendimento e esforço dos atletas; 7) feedback negativo (5 itens): comportamentos de punição e castigo tendo em vista gerir ou controlar os comportamentos inadequados dos atletas; 8) gestão partilhada do poder (6 itens): acções do treinador no sentido de promover um maior envolvimento dos atletas no processo de tomada de decisão, nomeadamente em aspectos relacionados com o treino e a competição; e 9) gestão passiva do poder (6 itens): evitamento na tomada de decisão e distanciamento dos técnicos em face das suas responsabilidades quando é necessário resolver problemas importantes. Os itens foram respondidos numa escala tipo Likert com cinco opções de resposta (1=Discordo totalmente; 5=Concordo totalmente). Dados da validade e utilização desse instrumento 
podem ser obtidos em Gomes (2005; 2008a). Os escores de cada dimensão são calculados por meio da soma dos itens correspondentes a cada subescala, dividindo-se depois esse valor pelo número total de itens. Os valores mais altos em cada subescala significam maiores frequências de comportamentos, por parte do treinador, em cada uma das áreas de acção descritas.

Questionário de Satisfação em Atletas (QSA) (Gomes, 2008c). Este instrumento é uma versão traduzida do Atblete Satisfaction Questionnaire (ASQ), desenvolvido por Riemer e Chelladurai (1998), encontrando-se em fase de validação para a população desportiva portuguesa. Para efeitos do presente estudo foram utilizadas quatro das subescalas do questionário relativas à satisfação dos atletas com a liderança, com um total de 19 itens: 1) Satisfação com a utilização das capacidades (5 itens): descreve a opinião dos atletas acerca do modo como o treinador utiliza e/ou maximiza as capacidades e o talento individual dos atletas; 2) Satisfação com a estratégia (6 itens): refere-se à avaliação que os atletas fazem acerca das estratégias e tácticas seguidas pelo treinador durante as competições; 3) Satisfação com o tratamento pessoal (5 itens): aponta a satisfação dos atletas com o tratamento dado pelo treinador que afecta cada um directamente e a equipa indirectamente, incluindo o apoio social e o feedback positivo; 4) Satisfação com o treino e instrução (3 itens): refere-se à satisfação dos atletas acerca do treino e instruções fornecidas pelo treinador. Os itens foram respondidos numa escala tipo Likert de 7 pontos (1=Mesmo nada satisfeito; $7=$ Extremamente satisfeito), sendo os escores calculados mediante a soma dos valores dos itens em cada uma das dimensões, dividindo, depois, esse valor pelo número total de itens de cada subescala. As pontuações mais altas significam valores mais elevados de satisfação em cada uma das dimensões avaliadas.

\section{Procedimento}

Numa primeira etapa, contactámos os treinadores responsáveis pelas diferentes equipas tendo em vista apresentar os objectivos do estudo e solicitar a autorização para a participação dos respectivos atletas. Após a concordância dos mesmos, apresentámos aos atletas os objectivos da avaliação a realizar. Para tal, foram obtidos os consentimentos informados dos atletas e respectivos pais, no caso dos praticantes serem menores de idade. A recolha dos instrumentos foi sempre efectuada de modo isolado à presença do treinador, dada a temática em estudo. A recolha dos protocolos de avaliação foi efectuada no próprio local de prática dos atletas. Assim, foram distribuídos 91 protocolos de avaliação, tendo sido considerados como válidos todos os questionários aplicados (taxa de retorno de 100\%).

\section{Resultados}

O tratamento e análise estatística dos dados foram efectuados no programa SPSS (versão 17.0 para Windows) e incluiu vários procedimentos que serão explicados no decorrer desta parte do trabalho.

\section{Estatísticas descritivas das variáveis em análise}

A Tabela 1 começa por fornecer indicação dos valores médios e descritivos das variáveis em análise. Como se poderá verificar, no caso da escala de liderança, as dimensões mais percepcionadas pelos atletas referiram-se ao treino e instrução e ao respeito e tratamento justo, enquanto as menos percepcionadas foram ambas as dimensões da gestão do poder. Já no caso do questionário de satisfação, foram as facetas da satisfação com o treino e instrução e da satisfação com a estratégia que obtiveram valores médios mais elevados. No caso da compatibilidade, observaram-se valores no terço superior da escala Likert, o que poderá sugerir níveis muito aceitáveis de identificação entre os atletas e os respectivos treinadores. De igual modo, verificámos os valores de assimetria e curtose para as dimensões em causa, não se excedendo os valores de cut-off de dois para ambos os indicadores, considerado como valor razoável de distribuição (West, Finch \& Curran, 1995).

Associagões entre as variáveis e indices de fidelidade das subescalas

Começando pelas associações e correlações existentes entre todas as variáveis em estudo, estas foram realizadas por meio do cálculo dos coeficientes de correlação de Pearson, descrevendo-se os valores encontrados na Tabela 2. De referir que esta análise foi efectuada separadamente para cada um dos grupos de atletas (novatos e experientes), apresentando-se igualmente os valores de correlação para a amostra total.

Assim, no que se refere às associações entre a compatibilidade treinador-atleta e as restantes dimensões, observaram-se associações positivas com algumas dimensões da liderança (e.g., treino e instrução, respeito e tratamento justo, visão de futuro e optimismo e motivação para a realização). No caso das equipas de atletas novatos, também se registou uma associação positiva com o feedback positivo e uma associação negativa com o feedback negativo. Por outro lado, constatou-se uma associação positiva entre a compatibilidade e a satisfação com a liderança, nos quatro domínios avaliados.

Quanto às subescalas do instrumento de 
avaliação da liderança, verificaram-se em geral as associações esperadas, ou seja, as dimensões mais "desejáveis" das acções dos treinadores (e.g., treino e instrução, respeito e tratamento justo, apoio social, visão de futuro e optimismo, motivação para a realização, feedback positivo e, em parte, a gestão partilhada do poder) relacionaram-se positivamente entre si, o que significa que aumentos em qualquer uma das dimensões repercutiram-se em percepções mais elevadas nas restantes facetas.

Menos evidentes foram as associações com a gestão passiva do poder e com o feedback negativo. Assim, no caso da gestão passiva verificaram-se valores significativos apenas nas equipas de atletas novatos, assumindo associações positivas com o apoio social, o feedback positivo e a gestão partilhada do poder. Quanto ao feedback negativo, observou-se apenas uma associação significativa nas equipas de atletas novatos, constatando-se que o uso deste comportamento se relacionou com uma menor percepção de respeito e tratamento justo.

No que se refere à relação entre a liderança e a satisfação, observou-se que as dimensões "positivas" da EMLD-2 associaram-se quase todas de um modo positivo com as quatro subescalas do QSA. Já os aumentos na gestão passiva do poder associaram-se a uma menor satisfação com o treino e instrução no grupo de novatos.

Ainda na Tabela 2, são apresentados os indicadores de fidelidade das subescalas para o total da amostra (alfa de Cronbach), podendo verificar-se níveis muito aceitáveis em praticamente todas as dimensões dos instrumentos utilizados (Nunnally \& Bernstein, 1994). A única excepção prendeu-se com o feedback negativo $(\alpha=0,67)$ tendo, no entanto, sido mantido nos estudo devido à sua proximidade do valor aceitável de 0,70 .

Tabela 1 - Estatísticas descritivas das variáveis em análise (amostra total)

\begin{tabular}{|c|c|c|c|c|c|c|}
\hline Variável & $\begin{array}{c}\text { Média e } \\
\text { (Desvio padrão) } \\
\end{array}$ & $\frac{\text { Erro padrão }}{\underline{\text { da medida }}}$ & $\begin{array}{c}\text { Valor } \\
\text { mínimo } \\
\end{array}$ & $\begin{array}{l}\text { Valor } \\
\text { máximo } \\
\end{array}$ & $\begin{array}{c}\text { Assimetria } \\
\text { (Erro padrão) } \\
\end{array}$ & $\begin{array}{c}\text { Curtose } \\
\text { (Erro padrão) } \\
\end{array}$ \\
\hline Compatibilidade & & & & & & \\
\hline MCTA: Total & $6,24(1,32)$ & 0,14 & 2,60 & 8,40 & $-0,64 \quad(0,25)$ & $-0,27 \quad(0,50)$ \\
\hline Liderança & & & & & & \\
\hline $\begin{array}{l}\text { EMLD-2: Treino e instrução } \\
\text { EMLD-2: Respeito e tratamento }\end{array}$ & $3,83(0,60)$ & 0,06 & 1,89 & 4,89 & $-0,72(0,25)$ & $0,34(0,50)$ \\
\hline 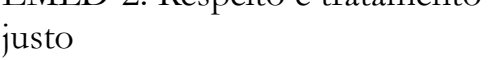 & $3,72(0,70)$ & 0,07 & 1,83 & 5,00 & $-0,36 \quad(0,25)$ & $0,04(0,50)$ \\
\hline EMLD-2: Apoio social & $2,49(0,70)$ & 0,07 & 1,00 & 4,00 & $0,20(0,25)$ & $-0,78(0,50)$ \\
\hline $\begin{array}{l}\text { EMLD-2: Visão de futuro e } \\
\text { optimismo }\end{array}$ & $3,53(0,61)$ & 0,06 & 2,00 & 4,60 & $-0,42(0,25)$ & $-0,62(0,50)$ \\
\hline $\begin{array}{l}\text { EMLD-2: Motivação para } \\
\text { realização }\end{array}$ & $3,63(0,58)$ & 0,06 & 2,00 & 4,67 & $-0,49 \quad(0,25)$ & $-0,40 \quad(0,50)$ \\
\hline EMLD-2: Feedback positivo & $3,35(0,66)$ & 0,07 & 1,67 & 4,83 & $-0,18(0,25)$ & $-0,22(0,50)$ \\
\hline EMLD-2: Feedback negativo & $3,53(0,62)$ & 0,07 & 1,60 & 5,00 & $-0,04 \quad(0,25)$ & $0,57(0,50)$ \\
\hline $\begin{array}{l}\text { EMLD-2: Gestão partilhada do } \\
\text { poder }\end{array}$ & $2,22(0,77)$ & 0,08 & 1,00 & 4,17 & $0,34(0,25)$ & $-0,57(0,50)$ \\
\hline $\begin{array}{l}\text { EMLD-2: Gestão passiva do } \\
\text { poder }\end{array}$ & $2,22(0,65)$ & 0,07 & 1,00 & 3,67 & $0,27(0,25)$ & $-0,66(0,50)$ \\
\hline $\begin{array}{l}\text { Satisfação } \\
\text { QSA: Satisfação com utilização }\end{array}$ & & & & & & \\
\hline de capacidades & $4,88(1,09)$ & 01 & 1,60 & 6,60 & $-0,49 \quad(0,25)$ & $-0,31(0,50)$ \\
\hline $\begin{array}{l}\text { QSA: Satisfação com a estratégia } \\
\text { QSA: Satisfação com o }\end{array}$ & $5,11(1,09)$ & 0,11 & 2,33 & 6,83 & $-0,41 \quad(0,25)$ & $-0,58(0,50)$ \\
\hline $\begin{array}{l}\text { tratamento pessoal } \\
\text { QSA: Satisfação com o treino e }\end{array}$ & $4,96(1,10)$ & 0,12 & 1,40 & 7,00 & $-0,63(0,25)$ & $0,36(0,50)$ \\
\hline instrução & $5,26(1,25)$ & 0,13 & 2,00 & 7,00 & $-0,58(0,25)$ & $-0,67 \quad(0,50)$ \\
\hline
\end{tabular}




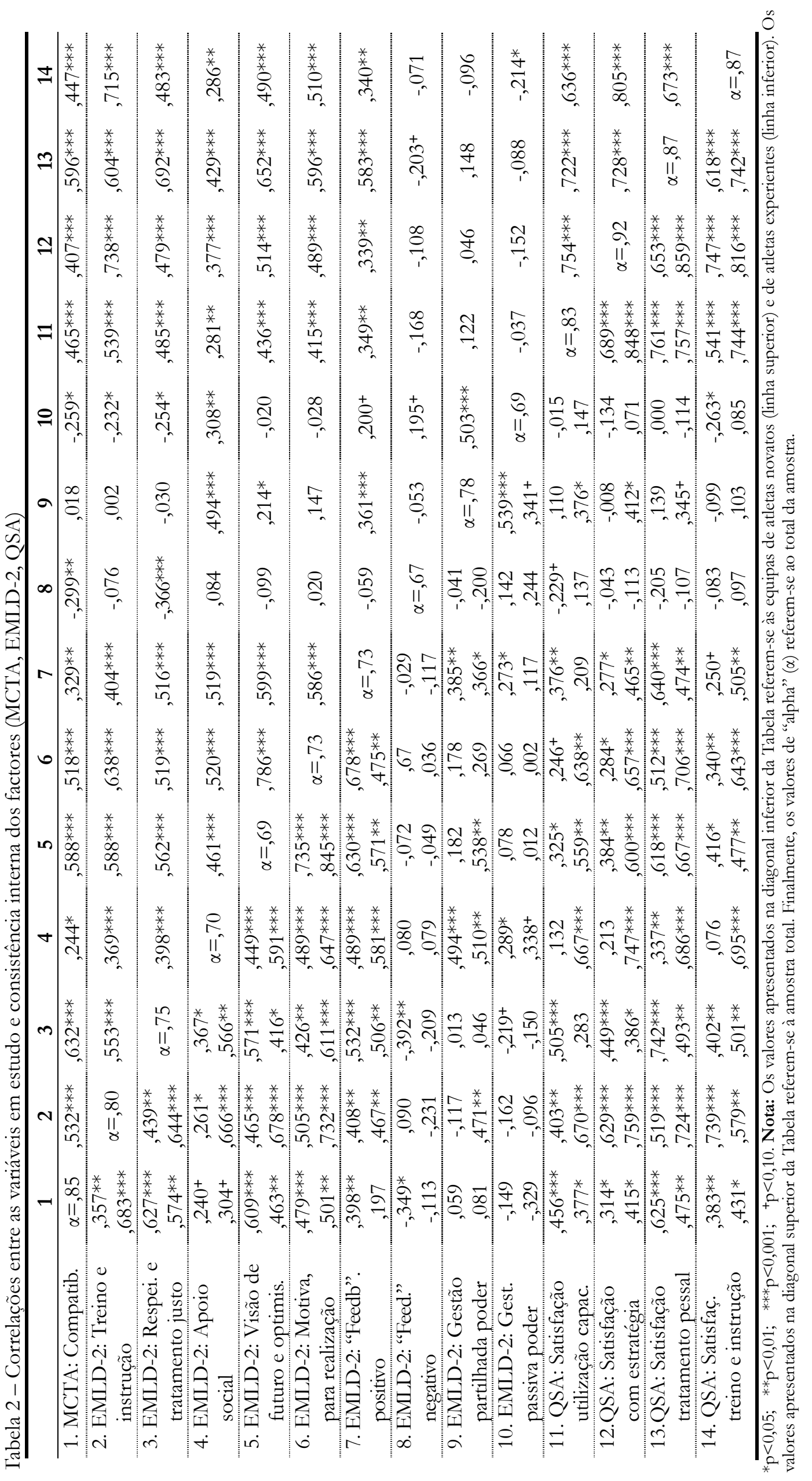

Psico-USF, v. 15, n. 2, p. 235-248, maio/ago. 2010 
Diferenças entre os grupos de novatos e experientes nas variáveis em estudo

Num segundo conjunto de análises, procurámos encontrar diferenças entre as equipas em análise (novatos e experientes) na percepção acerca dos estilos de liderança, compatibilidade e satisfação. Com o objetivo de permitir uma análise geral dos valores médios das variáveis em comparação em ambos os grupos de equipas, a Tabela 3 apresenta as estatísticas descritivas em todos os instrumentos aplicados.

Antes de apresentarmos os resultados nos dois grupos em comparação, devemos salientar que efectuámos uma análise exploratória aos dados, de modo a observar os pressupostos de aplicação dos testes paramétricos. Nesse sentido, para todas as análises realizadas testámos as diferenças, utilizando os testes paramétricos e não-paramétricos correspondentes, sempre que os pressupostos da normalidade não estavam garantidos, seguindo-se assim as indicações de Fife Schaw (2006). De um modo geral, os resultados comparativos foram similares, tendo sido por isso nossa decisão apresentar os resultados dos testes paramétricos, uma vez que estes são mais robustos e nos permitem usar análises multivariadas, as quais têm como vantagem a redução do número de testes a executar, diminuindo assim a probabilidade do erro Tipo 1.

Assim, no que se refere à medida de compatibilidade, as análises de variância unifactorial revelaram-se significativas $(F(1,89)=5,19 ; \mathrm{p}<0,05)$. De acordo com os valores médios, verificou-se que as equipas de novatos atribuíram maiores valores de compatibilidade com os respectivos treinadores (Tabela 3).

Quanto aos estilos de liderança, foram verificadas diferenças estatisticamente significativas nas dimensões de liderança (Wilks' lambda=0,71, F(9), 81) $=3,68, \quad \mathrm{p}<0,01) . \quad$ Os testes univariados demonstraram uma tendência dos atletas novatos assinalarem aos respectivos treinadores maiores níveis de treino e instrução, respeito e tratamento justo, visão de futuro e optimismo, motivação para a realização e, inversamente, menor gestão passiva do poder (Tabela 3).

No que se refere às medidas de satisfação, encontrámos também valores significativos entre os grupos em estudo (Wilks' lambda $=0,83, \mathrm{~F}(4,86)=4,32$, $\mathrm{p}<0,01)$. Os testes univariados demonstraram uma tendência dos atletas novatos experienciarem maior satisfação nas quatro dimensões avaliadas pelo QSA (Tabela 3).

Tabela 3 - Média, desvio padrão e valores dos testes univariados na compatibilidade, liderança e satisfação: diferenças em função das variáveis demográficas e desportivas

\begin{tabular}{|c|c|c|c|}
\hline Variável & $\begin{array}{c}\text { Novatos }(\mathrm{n}=59) \\
\mathrm{M}(\mathrm{DP})\end{array}$ & $\begin{array}{c}\text { Experientes }(n=32) \\
M(D P)\end{array}$ & $\mathrm{F}$ \\
\hline \multicolumn{4}{|l|}{ Compatibilidade } \\
\hline MCTA: Total & $6,46(1,28)$ & $5,81 \quad(1,31)$ & $5,19 *(1,89)$ \\
\hline \multicolumn{4}{|l|}{ Liderança } \\
\hline EMLD-2: Treino e instrução & $4,02(0,47)$ & $3,47(0,64)$ & $22,85^{* * *}(9,81)$ \\
\hline EMLD-2: Respeito e tratamento justo & $3,86(0,72)$ & $3,46(0,59)$ & $6,95^{*}$ \\
\hline EMLD-2: Apoio social & $2,46(0,67)$ & $2,54(0,75)$ & 0,24 \\
\hline EMLD-2: Visão de futuro e optimismo & $3,65(0,61)$ & $3,29(0,55)$ & $7,60 * *$ \\
\hline EMLD-2: Motivação para realização & $3,75(0,54)$ & $3,43(0,61)$ & $6,42^{*}$ \\
\hline EMLD-2: Feedback positivo & $3,37(0,66)$ & $3,30(0,66)$ & 0,22 \\
\hline EMLD-2: Feedback negativo & $3,48(0,68)$ & $3,64(0,49)$ & 1,53 \\
\hline EMLD-2: Gestão partilhada do poder & $2,12(0,80)$ & $2,42(0,66)$ & $3,28^{+}$ \\
\hline EMLD-2: Gestão passiva do poder & $2,09(0,63)$ & $2,44(0,61)$ & $6,42^{*}$ \\
\hline \multicolumn{4}{|l|}{ Satisfação } \\
\hline QSA: Satisfação com utilização de capacidades & $5,09(1,12)$ & $4,49(0,93)$ & $6,63 *(4,86)$ \\
\hline QSA: Satisfação com a estratégia & $5,42(0,96)$ & $4,54(1,10)$ & $15,41 * * *$ \\
\hline QSA: Satisfação com o tratamento pessoal & $5,13(1,14)$ & $4,64(0,97)$ & $4,21 *$ \\
\hline QSA: Satisfação com o treino e instrução & $5,58(1,13)$ & $4,66(1,27)$ & $12,78^{* *}$ \\
\hline
\end{tabular}
${ }^{*} \mathrm{p}<0,05 ; \quad{ }^{*} \mathrm{p}<0,01 ; \quad{ }^{*} *{ }^{*} \mathrm{p}<0,001 ;{ }^{+} \mathrm{p}<0,10$ 


\section{Predição da compatibilidade treinador-atleta}

No último tipo de análises realizadas, procurámos observar quais as dimensões da liderança (EMLD-2) que melhor poderiam predizer a compatibilidade treinador-atleta (MCTA). Ou seja, neste caso interessava perceber quais as facetas da liderança que melhor poderiam explicar a identificação pessoal e desportiva entre atletas e respectivos treinadores. Para tal, efectuámos análises de regressão hierárquica (método "enter"), apresentando-se igualmente alguns indicadores de multicolinearidade (indices de tolerância-IT, variance inflaction factor - VIF e o condition index - $\mathrm{CI}$ ), constatando-se em geral a ausência deste problema nos modelos testados. De salientar ainda que a predição da compatibilidade foi realizada separadamente em cada um dos grupos estudados (novatos e experientes).

Começando pelas equipas de formação (novatos), as dimensões "número de anos com o actual treinador" e "títulos alcançados com o actual treinador" apresentaram associações positivas com a compatibilidade. Convém realçar que, no primeiro caso dividimos a amostra entre atletas a trabalhar o primeiro ano com o respectivo treinador e atletas com mais de um ano de experiência com os treinadores. No segundo caso, dividimos a amostra entre atletas que obtiveram títulos desportivos de relevo em nível nacional com os respectivos treinadores (e.g., campeões nacionais e vencedores da Taça de Portugal) e atletas sem esses mesmos registos. Assim, no modelo de regressão a variável "número de anos com o actual treinador" foi introduzida no primeiro bloco de entrada seguida da variável "títulos alcançados com o actual treinador". No primeiro bloco de entrada, foram encontradas diferenças significativas $(F(1,57)=7,02$, $\mathrm{p}<0,05)$ explicando-se $9 \%$ da variância. Neste caso, atletas com menos anos de trabalho com o treinador assumiram maior compatibilidade com o treinador $(\beta=-0,33, \quad t=-2,65, \quad \mathrm{p}<0,05) \quad(\mathrm{IT}=0,54 ; \quad \mathrm{VIF}=1,87 ;$ $\mathrm{CI}=2,61)$. De seguida controlaram-se os títulos alcançados com o actual treinador, passando o modelo a predizer $19 \%$ da variância $(F(2,56)=7,77, \mathrm{p}<0,05)$. Atletas com registos desportivos de relevo assumiram maiores níveis de compatibilidade com o actual treinador $\quad(\beta=-0,41, \quad t=-2,78, \quad \mathrm{p}<0,01) \quad(\mathrm{IT}=0,45$; $\mathrm{VIF}=2,20 ; \quad \mathrm{CI}=9,00)$. A adição das dimensões de liderança no terceiro bloco resultou igualmente num modelo significativo $(F(5, \quad 53)=13,07, \quad \mathrm{p}<0,001)$ explicando $51 \%$ da variância. Após controlar os efeitos das variáveis número de anos com o actual treinador e títulos alcançados com o actual treinador, emergiram como preditores significativos a visão de futuro e optimismo e, com valores praticamente significativos, o feedback negativo (neste último caso o valor de $\mathrm{p}$ foi igual a 0,054, sendo, por isso, marginalmente significativo). Assim sendo, a compatibilidade treinador-atleta foi predita pela maior visão de futuro e optimismo $\quad(\beta=0,49, \quad t=4,04, \quad \mathrm{p}<0,001) \quad(\mathrm{IT}=0,057$;
$\mathrm{VIF}=1,76 ; \mathrm{CI}=23,3) \mathrm{e}$ pelo menor uso do feedback negativo $\quad(\beta=-0,21, \quad t=-1,97, \quad \mathrm{p}=0,054) \quad(\mathrm{IT}=0,74$; $\mathrm{VIF}=1,35 ; \mathrm{CI}=28,97)$.

No que respeita às equipas de competição (experientes), nenhuma das variáveis desportivas anteriores (e.g., número de anos com o actual treinador e títulos alcançados com o actual treinador) apresentou associações positivas com a compatibilidade. Nesse sentido, não foram introduzidas no modelo de regressão. Por isso, para a predição da compatibilidade treinador-atleta foram apenas incluídas as facetas da liderança que evidenciaram associações positivas com a medida de compatibilidade, obtendo-se um modelo significativo $(F(4,27)=6,82, \mathrm{p}<0,01)$. Assim sendo, no modelo final foram incluídas as dimensões de treino e instrução, respeito e tratamento justo, visão de futuro e optimismo e motivação para a realização, predizendo o modelo $43 \%$ da variância. No entanto, apenas a faceta de treino e instrução assumiu valores significativos no modelo final $(\beta=0,55, t=2,47, \mathrm{p}<0,05) \quad(\mathrm{IT}=0,37$; $\mathrm{VIF}=2,69 ; \mathrm{CI}=16,08)$. Nesse sentido, compatibilidade treinador-atleta foi predita pela maior utilização do treino e instrução por parte do treinador.

\section{Discussão}

O presente estudo teve como principal objectivo avaliar a relação treinador-atleta, incluindo as abordagens mais recentes no estudo da liderança. Nesse sentido, procurámos observar a forma como os treinadores são percepcionados pelos atletas e qual a importância de algumas variáveis pessoais e desportivas dos praticantes na explicação desse fenómeno.

Um primeiro aspecto a salientar prende-se com os valores médios das subescalas dos instrumentos de liderança (EMLD-2). Nesse caso, observou-se um padrão semelhante de resultados entre ambos os grupos de atletas, emergindo como dimensões fundamentais as relacionadas com a acção "transformacional" dos treinadores (e.g., treino e instrução, respeito e tratamento justo, motivação para a realização e visão de futuro e optimismo). Curiosamente, tanto as dimensões do feedback positivo como negativo, obtiveram valores médios semelhantes e acentuados, o que faz pressupor a sua utilização habitual no relacionamento com os atletas. Já com resultados inferiores, observaram-se as dimensões do apoio social e os domínios da gestão do poder que, curiosamente, foram mais baixos no grupo de atletas novatos. Seja como for, esses dados sugerem a importância das acções transformacionais dos treinadores no nível desportivo, confirmando-se assim a importância que têm vindo a adquirir noutros contextos sociais e profissionais (Bass, 1985; Bryman, 1992; Conger, 1989; Conger \& Kanungo, 1987; Sashkin, 1988).

Esses resultados médios das subescalas são 
também reforçados pelas associações entre as variáveis, uma vez que o principal aspecto a reter dos dados obtidos prende-se com a situação das facetas mais desejáveis da liderança, que se relacionaram positivamente com a compatibilidade com o treinador e com a satisfação com a liderança. Na prática, pode-se afirmar que uma maior tendência dos treinadores para a utilização do treino e instrução, do respeito e tratamento justo, do apoio social (embora nem todas as associações tenham sido significativas neste último caso), da visão de futuro e optimismo e da motivação para a realização, implicaram um sentimento de maior identificação pessoal e desportiva com os treinadores, bem como avaliações mais positivas acerca do modo como estes exercem o poder (ou seja, maior satisfação com a liderança). Esse aspecto é tão mais relevante pois os outros domínios avaliados pela EMLD-2 não evidenciaram o mesmo padrão de associações. Assim, tanto as dimensões do feedback negativo e positivo (mais próximas do conceito de liderança transaccional) como as dimensões da gestão do poder (mais próximas do conceito de tomada de decisão) não assumiram associações tão fortes $\mathrm{e} / \mathrm{ou}$ significativas com a compatibilidade com o treinador e com a satisfação com a liderança. Repare-se que no caso do feedback negativo as relações encontradas limitaram-se a apenas duas áreas (e.g., a compatibilidade entre treinador e atletas e o respeito e tratamento justo), sendo algo inesperada a ausência de correlação com o feedback positivo que, pelo contrário, apresentou maiores relações com as dimensões "transformacionais" da liderança. Deste ponto de vista, estas duas facetas da acção dos treinadores (feedback positivo e negativo) parecem independentes entre si, podendo estabelecerse um paralelismo entre este resultado e os encontrados por Bass e Avolio (1990; 1997) com a MLQ (Multifactor Leadership Questionnaire). Nesse caso, verificou-se que também existia uma tendência do reforço contingente (domínio muito semelhante ao feedback positivo) se associar mais às dimensões transformacionais da escala do que aos outros domínios transaccionais, nomeadamente à faceta de intervenção em crise (activa). Nesse mesmo sentido podem ser interpretados os resultados obtidos nas dimensões de gestão partilhada e passiva do poder. De facto, enquanto no caso da gestão partilhada as associações foram no sentido esperado para um ou ambos os grupos de atletas (e.g., relações positivas com o treino e instrução, o apoio social, a visão de futuro e optimismo e o feedback positivo), o mesmo não se poderá dizer da gestão passiva do poder, que assumiu igualmente associações positivas com o apoio social, o feedback positivo e a própria gestão partilhada do poder. Repare-se que este padrão de associações foi apenas significativo para o grupo de atletas novatos, podendo assim ser o factor idade e experiência dos atletas a explicar a razão de terem percebido no mesmo sentido uma faceta de liderança preconizada como negativa e outras três preconizadas como positivas. Nesse caso, será necessário aguardar por estudos futuros que possam ajudar a confirmar estes resultados.

Seja como for, de um modo geral, a relação desses dados com a literatura é relativamente evidente, uma vez que são vários os estudos que têm vindo a demonstrar que as acções mais positivas (e desejáveis) da acção dos treinadores (e.g., feedback positivo, treino e instrução, etc.) tendem a associar-se positivamente com a satisfação pessoal e desportiva dos atletas, bem como com a satisfação com a liderança dos treinadores (Dwyer \& Fischer, 1990; Schliesman, Beitel \& DeSensi, 1994). Disso mesmo nos deu conta Chelladurai, Imamura, Yamaguchi, Oinuma e Miyauchi, (1988), num estudo com atletas universitários do Canadá e do Japão, onde foi possível constatar associações positivas entre todas as dimensões da escala de liderança no desporto (exceptuando o comportamento autocrático) e a satisfação com a liderança.

No nosso caso, talvez o aspecto "inovador" seja a possibilidade dessas associações serem extensíveis às dimensões transformacionais das acções dos treinadores, o que sustenta a importância da sua incorporação na compreensão da liderança desportiva.

O segundo aspecto a reter deste estudo, referese à comparação entre grupos de atletas. Neste caso, ficou evidente a maior tendência dos atletas novatos assumirem uma maior compatibilidade com os treinadores, avaliando também mais positivamente os seus estilos de liderança (e.g., maiores níveis de treino e instrução, respeito e tratamento justo, visão de futuro e optimismo e motivação para a realização). Pelo lado inverso, esses atletas atribuíram menor tendência aos treinadores para a gestão passiva do poder do que os seus colegas experientes.

A comparação destes dados com a literatura é algo difícil, pela escassez de dados sobre esta temática. Em termos gerais, as indicações existentes realçam que os atletas, com o avançar dos anos, tendem a preferir estilos de liderança mais centrados no apoio social e na relação e menos comportamentos de feedback positivo, que tendem a ser mais preferidos pelos mais novos (Chelladurai \& Carron, 1983; Terry, 1984). Já no nível dos comportamentos centrados na tarefa (e.g., treino e instrução), os resultados são menos claros, mas representam uma das dimensões mais preferidas em todas as idades (Riemer, 2007). Seja como for, alguns estudos têm verificado algumas diferenças entre idades, observando-se avaliações mais positivas por parte dos atletas mais novos relativamente aos estilos de liderança (Gomes \& Machado, 2009), bem como a tendência para percepcionarem uma maior orientação para a relação por parte dos treinadores (Gomes, Pereira, \& Pinheiro, 2008).

O terceiro, e último resultado a considerar neste estudo, relaciona-se com a predição da compatibilidade treinador-atleta. No caso das equipas de atletas novatos, constituíram-se como variáveis preditoras a 
maior utilização da visão de futuro e optimismo por parte dos treinadores e, com valores quase significativos, a menor aplicação do feedback negativo. Assim, pode-se afirmar que o sentimento de proximidade dos atletas relativamente aos respectivos treinadores é predita pela maior capacidade desses últimos definirem uma visão de futuro interessante para os atletas, estimulando-os e encorajando-os nesse mesmo sentido (em paralelo, com baixa utilização de punições e comentários negativos). Também devemos salientar que duas variáveis desportivas concorreram na explicação deste fenómeno, verificando-se que a maior compatibilidade com o treinador foi também predita pelo menor número de anos de trabalho com o treinador e, como seria de esperar, pela existência de registos desportivos de relevo. Apesar dos resultados no número de anos de trabalho com o actual treinador parecer algo "contraditório", pois seria de esperar que o passar dos anos fosse um preditor da maior compatibilidade com o treinador, convém realçar que esta variável apresenta um espectro de tempo muito reduzido nessas idades, sendo habitual os atletas ficarem apenas um ou dois anos com o mesmo treinador nesses escalões desportivos, o que na prática inviabiliza a possibilidade de testar esse efeito ao longo do tempo. Quanto à possibilidade do maior sucesso desportivo predizer a maior compatibilidade com os treinadores nas equipas de atletas novatos, este aspecto está em linha com dados de outros estudos onde os praticantes com melhores registos competitivos tendem a avaliar mais positivamente os seus treinadores (Antunes \& Cruz, 1997; Gomes \& Cruz, 2006; Horn, 2008; Leitão, 1999; Riemer \& Chelladurai, 1995).

Já no grupo de atletas experientes, apesar de terem "entrado" inicialmente no modelo de regressão várias dimensões da EMLD-2, apenas o treino e instrução se constitui como variável preditora da compatibilidade com o treinador. Esse aspecto é tão mais relevante pela circunstância do modelo final ter explicado praticamente metade da variância associada a esse fenómeno. $\mathrm{Na}$ prática, os atletas mais velhos parecem sentir maior identificação com os respectivos treinadores à medida que estes se centram nos aspectos relacionados com as tarefas a realizar, dando indicações sobre aquilo que os atletas devem fazer em treino e competição e corrigindo eventuais erros cometidos.

Esses resultados fazem supor que os aspectos relacionados com a articulação de uma visão de futuro desafiante e motivadora por parte dos treinadores, para o grupo de atletas novatos, e a orientação para a tarefa, no caso dos treinadores de equipas de atletas experientes, explicam melhor a proximidade e identificação com os responsáveis técnicos. Essas diferenças podem ser compreendidas pelo factor idade, pois no caso dos mais novos será a perspectiva de desenvolvimento pessoal e a aprendizagem das competências da modalidade (idealizando-se o futuro enquanto atleta) que maior identificação poderá promover com os treinadores. Já nos mais velhos, são os aspectos relacionados com a execução das tarefas que mais tendem a interessar os atletas na relação com os respectivos treinadores. Curiosamente, alguns destes dados encontram relação nos estudos sobre a satisfação com a liderança em contextos organizacionais, onde certas facetas dos líderes como, por exemplo, a visão motivadora e inspiradora do trabalho, tendem a gerar níveis mais elevados de rendimento nos membros do grupo e a promover experiências laborais mais positivas nos colaboradores (Avolio \& Bass, 1988; Brown \& Dodd, 1999; Podsakoff, MacKenzie, Moorman \& Fetter, 1990). Já em contextos desportivos, um estudo de Rowold (2006) com praticantes de artes marciais, constatou que as dimensões transformacionais da acção dos treinadores (e.g., motivação inspiradora, influência nos ideais e consideração individual) contribuíam de um modo significativo para explicar a eficácia dos treinadores. Deste ponto de vista, também a EMLD-2 poderá ajudar a analisar até que ponto as acções dos treinadores predizem dimensões tão importantes como a satisfação, coesão e rendimento dos atletas e equipas.

\section{Considerações finais}

Este estudo sugere a importância da liderança transformacional na acção dos treinadores em ambos os grupos de idades, sendo de destacar que os atletas mais novos avaliaram mais positivamente os seus treinadores e assumiram maior compatibilidade com os respectivos técnicos. Estes dados são congruentes com a satisfação com a liderança, uma vez que o grupo de atletas novatos evidenciou avaliações mais positivas acerca da liderança dos responsáveis técnicos. Paralelamente, constatou-se que a articulação de uma visão de futuro e o consequente encorajamento das capacidades dos atletas por parte dos treinadores parecem particularmente indicadas para os praticantes mais novos, enquanto a concentração na realização da tarefa parece mais adequada para os praticantes mais velhos.

Quanto a limitações deste estudo, talvez o aspecto mais saliente diga respeito à circunstância de termos na amostra apenas atletas de sexo masculino, fazendo por isso sentido analisar este fenómeno junto da população feminina. Nesse sentido, será da congregação dos esforços para alargarmos o estudo dos factores relacionados com a eficácia dos comportamentos dos treinadores que melhor poderemos explicar cientificamente o seu papel no fenómeno desportivo.

\section{Referências}

Adelino, J., Vieira, J. \& Coelho, O. (2000). Treino de 
jovens: o que todos precisam de saber. Lisboa: Centro de Estudos e Formação Desportiva.

Antunes, J. M. \& Cruz, J. F. (1997). Dinâmica de grupos e sucesso na alta competição: as relações entre liderança, coesão, satisfação e rendimento no basquetebol. Em JF. Cruz \& A.R. Gomes (Eds.). Psicologia aplicada ao desporto e à actividade física: teoria, investigação e intervenção (pp. 141-169). Braga: Universidade do Minho-APPORT.

Avolio, B. J. \& Bass, B. M. (1988). Transformational leadership, charisma and beyond. Em J.G. Hunt, H.R. Baliga, H.P. Dachler \& C.A. Schriesheim (Eds.). Emerging leadership vistas (pp. 29-49). Lexington, MA: Heath.

Bass, B. M. (1985). Leadership and performance beyond expectations. Nova Iorque: The Free Press.

Bass, B. M. (1990). Bass and Stogdill's handbook of leadership ( $3^{\mathrm{a}}$ ed.). Nova Iorque: Free Press.

Bass, B. M. \& Avolio, B. J. (1990). Transformational leadership development: manual for the multifactor leadership questionnaire. Palo Alto, CA: Consulting Psychologists Press.

Bass, B. M. \& Avolio, B. J. (1997). Full Range leadership development: manual for the Multifactor Leadership Questionnaire. Califórnia: Mind Garden.

Bloom, B. S. (1985). Developing talent in young people. Nova Iorque: Ballantine.

Brown, F. W. \& Dodd, N. G. (1999). Rally the troops or make the trains run on time: the relative importance and interaction of contingent reward and transformational leadership. Leadership \& Organization Development Journal, 20, 291-299.

Bryman, A. (1992). Charisma and leadership in organizations. Londres: Sage.

Chelladurai, P. (1984a). Leadership in sports. Em J.M. Silva \& R.S. Weinberg (Eds.). Psychological foundations of sport (pp. 329-339). Champaign, IL: Human Kinetics.

Chelladurai, P. (1984b). Discrepancy between preferences and perceptions of leadership behavior and satisfaction of athletes in varying sports. Journal of Sport Psychology, 6, 27-41.

Chelladurai, P. (1993). Leadership. In R. N. Singer, M. Murphey \& L. K. Tennant (Eds.), Handbook of research on sport psychology (pp. 647-671). New York: Macmillan.

Chelladurai, P. (2007). Leadership in sports. Em G. Tenenbaum \& R. C. Eklund (Eds.). Handbook of Sport Psychology (pp. 113-135). Hoboken, New Jersey: John Wiley \& Sons.

Chelladurai, P. \& Carron, A. V. (1983). Athletic maturity and preferred leadership. Journal of Sport Psychology, 5, 371-380.

Chelladurai, P. \& Riemer, H. A. (1997). A classification of facets of athletic satisfaction. Journal of Sport Management, 11, 133-159.

Chelladurai, P. \& Saleh, S. D. (1978). Preferred leadership in sports. Canadian Journal of Applied Sport Sciences, 3, 85-92.

Chelladurai, P. \& Saleh, S. D. (1980). Dimensions of leader behavior in sports: development of a leadership scale. Journal of Sport Psychology, 2, 3445.

Chelladurai, P., Imamura, H., Yamaguchi, Y., Oinuma, Y. \& Miyauchi, T. (1988). Sport leadership in a cross-national setting: the case of Japanese and Canadian university athletes. Journal of Sport \& Exercise Psychology, 10, 374-389.

Conger, J. A. (1989). The charismatic leader: Behind the mystic of exceptional leadership. São Francisco: JosseyBass.

Conger, J. A. \& Kanungo, R. (1987). Toward a behavioural theory of charismatic leadership in organizational settings. The Academy of Management Review, 12, 637-647.

Côté, J. (1999). The influence of the family in the development of talent in sport. The Sport Psychologist, 13, 395-417.

Côté, J. \& Hay, J. (2002). Children's involvement in sport: a developmental perspective. Em J.M. Silva \& D.E. Stevens (Eds.). Psychological foundations of sport (pp. 484-502). Boston: Allyn and Bacon.

Côté, J., Young, B. W., Duffy, P. \& North, J. (2007). Towards a definition of excellence in sport coaching. International Journal of Coaching Science, 1, 3-17

Dwyer, J. M. \& Fischer, D G. (1990). Wrestler's perceptions of coaches' leadership as predictors of satisfaction with leadership. Perceptual \& Motor Skills, 71, 511-517.

Fife-Schaw, C. (2006). Levels of measurement. Em G. M. Breakwell, S. Hammond, C. Fife-Schaw \& J.A. Smith (Eds.). Research methods in psychology ( $3^{\mathrm{a}}$ ed.) (pp. 50-63). London: Sage.

Gardner, D. E., Shields, D. L, Bredmeier, B. J. \& Bostrom, A. (1996). The relationship between perceived coaching behaviors and team cohesion among baseball and softball players. The Sport Psychologist, 10, 367-381.

Gomes, A. R. (1997). Aspectos psicológicos da iniciação e formação desportiva: O papel dos pais. Em J.F. Cruz \& A.R. Gomes (Eds.). Psicologia aplicada ao desporto e actividade fisica: teoria, investigação 
e intervenção (pp. 291-319). Braga: APPORT (Associação dos Psicólogos Portugueses) Universidade do Minho. Obtido do World Wide Web: <http://hdl.handle.net/1822/5337>.

Gomes, A. R. (2005). Liderança e relação treinador-atleta em contextos desportivos. Tese de Doutoramento. Braga: Universidade do Minho. Obtido do World Wide Web: <http://hdl.handle.net/1822/3334>.

Gomes, A. R. (2008a). Escala Multidimensional de Liderança no Desporto: reanálise da estrutura factorial. Em A. P. Noronha, C. Machado, L. Almeida, M. Gonçalves, \& V. Ramalho (Eds.). Actas da XIII conferência internacional de avaliação psicológica: Formas e contextos. Braga: Psiquilíbrios Edições. Obtido do World Wide Web: <http://hdl.handle.net/1822/8296>.

Gomes, A. R. (2008b). Medida de Compatibilidade Treinador-Atleta (MCTA). Relatório técnico não publicado. Braga: Universidade do Minho.

Gomes, A. R. (2008c). Questionário de Satisfação em Atletas (QSA). Relatório técnico não publicado. Braga: Universidade do Minho.

Gomes, A. R., \& Cruz, J. F. (2006). Estilos de liderança, coesão e satisfação no desporto: análise das diferenças em função do tipo de modalidade praticada e do sucesso desportivo obtido pelos atletas. Em N. Santos, M. Lima, M. Melo, A. Candeias, M. Grácio \& A. Calado (Eds.). Actas do VI simpósio nacional de investigação em psicologia (vol. 11, pp. 103-123). Évora: Departamento de Psicologia, Universidade de Évora. Obtido do World Wide Web: <http://hdl.handle.net/1822/5985>.

Gomes, A. R. \& Machado, A. A. (2009). Liderança, coesão e satisfação em equipas de voleibol portuguesas: indicações da investigação e implicações práticas. Trabalho submetido para publicação.

Gomes, A. R., Pereira, A. P. \& Pinheiro, A. R. (2008). Liderança, coesão e satisfação em equipas desportivas: um estudo com atletas portugueses de futebol e futsal. Psicologia: Reflexão e Crítica, 21(3), 482-491.

Horn, T. S. (2008). Advances in sport psychology ( $3^{\mathrm{a}}$ ed.). Champaign, IL: Human Kinetics.

Horne, T. \& Carron, A. V. (1985). Compatibility in coach-athlete relationships. Journal of Sport Psychology, 7, 137-149.

Kenow, L. J. \& Williams, J. M. (1999). Coach-athlete compatibility and athlete's perception and evaluative reactions to coaching behaviors. Journal of Sport Behavior, 22, 251-259.

Leitão, J. C. (1999). A relação treinador-atleta: percepção dos comportamentos de liderança e de coesão em equipas de futebol. Tese de Doutoramento. Coimbra: Universidade de Coimbra.

Martens, R. (1990). Successful coaching. Champaign, IL: Human Kinetics.

Martens, R. (1996). Turning kids on the physical activity for a lifetime. Quest, 48, 303-310.

Nunnally, J. C. \& Bernstein, I. H. (1994). (3 ${ }^{\text {a }}$ ed.) Psychometric theory. Nova Iorque: McGraw Hill.

Pease, D. G. \& Kozub, S. A. (1994). Perceived coaching behaviors and team cohesion in high school girls basketball teams. Journal of Sport \& Exercise Psychology, 16, S93.

Podsakoff, P. M., MacKenzie, S. B., Moorman, R. H. \& Fetter, R. (1990). Transformational leader behaviors and their effects on followers' trust in leader, satisfaction, and organizational citizenship behaviors. Leadership Quarterly, 1, 107-142.

Riemer, H. A. (2007). Multidimensional model of coach leadership. Em S. Jowett \& D. Lavallee (Eds.). Social Psychology in sport (pp. 57-73). Champaign, IL: Human Kinetics.

Riemer, H. A. \& Chelladurai, P. (1995). Leadership and satisfaction in athletics. Journal of Sport \& Exercise Psychology, 17, 276-293.

Riemer, H. A. \& Chelladurai, P. (1998). Development of the athlete satisfaction questionnaire (ASQ). Journal of Sport and Exercise Psychology, 20, 127-156.

Rowold, J. (2006). Transformational and transactional leadership in martial arts. Journal of Applied Sport Psychology, 18, 312-325.

Saal, F. E. \& Knight, P. A. (1988). Industrial/organizational psychology: science and practice. Pacific Grove, CA: Brooks/Cole.

Sashkin, M. (1988). The visionary leader. Em J.A. Conger \& R.N. Kanungo (Eds.). Charismatic leadership: the elusive factor in organizational effectiveness (pp. 122-160). São Francisco: Jossey-Bass.

Schliesman, E. S., Beitel, P.A. \& DeSensi, J. T. (1994). Athlete and coach gender, leader behavior, and follower satisfaction in sport. Unpublished manuscript, University of Tennessee Knoxville, TN.

Terry, P. C. (1984). The coaching preferences of elite athletes competing at Universiade'83. Canadian Journal of Applied Sport Sciences, 9, 201-208.

Weinberg, R. S. \& Gould, D. (2007). Foundations of sport and exercise psychology ( $4^{\mathrm{a}}$ ed.). Champaign, IL: Human Kinetics.

Weiss, M. R. \& Friedrichs, W. D. (1986). The influence of leader behaviors, coach attributes, and institutional variables on performance and 
satisfaction of collegiate basketball teams. Journal of Sport Psychology, 8, 332-346.

West, S. G., Finch, J. F. \& Curran, P. J. (1995). Structural equation models with nonnormal variables: Problems and remedies. Em R.H. Hoyle (Ed.). Structural equation modeling: concepts, issues and applications (pp. 56-75). Newbury Park: Sage.

Westre, K. \& Weiss, M. (1991). The relationship between perceived coaching behaviors and group cohesion in high school football teams. The Sport Psychologist, 5, 41-54.
Williams, J. M., Jerome, G. J., Kenow, L. J., Rogers, T., Sartain, T. A. \& Darland, G. (2003). Factor structure of the coaching behavior questionnaire and its relationships to athlete variables. The Sport Psychologist, 17, 16-34.

Sobre os autores:

Recebido em novembro de 2009 Reformulado em fevereiro de 2010 Aprovado em junho de 2010

Rui Gomes obteve os diferentes graus académicos (Licenciatura, Mestrado e Doutoramento) na Área da Psicologia, pela Universidade do Minho, Braga. Ao longo da carreira profissional, trabalhou como psicólogo desportivo em clubes de diferentes modalidades. Actualmente, exerce funções como prof. auxiliar na Escola de Psicologia da Universidade do Minho e é investigador nas áreas da psicologia do desporto e do stresse ocupacional.

Paulo Paiva obteve os diferentes graus académicos (Licenciatura e Mestrado) na Área da Psicologia (especialização em Psicologia do Desporto), pela Universidade do Minho, Braga. Actualmente, exerce funções como psicólogo em equipas de formação desportiva no futebol e tem interesses de investigação no domínio da liderança e relação treinador-atleta. 